\title{
Transition Curvature of Soft Matrix Composite Laminates during Bending
}

\author{
Z.Y. Xiong ${ }^{1, a}$, R. X. Song ${ }^{1}$, R. Wu ${ }^{1}$, Z.X. Kang ${ }^{1}$, Q. Liu ${ }^{1}$ and H.J. Zhang ${ }^{1}$ \\ ${ }^{1}$ Beijing Municipal Institute of Labour Protection, Beijing 100054, China
}

\begin{abstract}
As an Elastic memory composite (EMC) laminate is bent, the prebuckled plate theory is valid until the transition curvature at which point the postbuckled solution becomes effective. This is the point at which microbuckling is predicted to occur. From the point onward, EMC laminate follows the postbuckled solution. In this paper, a solution of the transition curvature is proposed by combining matrix shear energy with Timoshenko's elastic foundation energy. By comparing with the intersection point of the straight line given by the prebuckled plate theory and the curve given by the postbuckled solution, the solution can be used to predict the point at which microbuckling is predicted to occur.
\end{abstract}

\section{Introduction}

Elastic memory composites (EMCs) are similar to the conventional fiber-reinforced composites except matrix material having a shape-memory function as compared with the conventional polymer matrix. Due to some valuable properties, such as high folding deformation, very high deployed stiffness- and strength-to-weight ratios, low density[1], EMCs have attracted considerable interests in the applications of deployable space-structure industry. Several research teams have reported successful fabrications of various components for deployable structures using EMCs, such as laminated plates and shells, pultruded rods, open-grid lattices, and hinges[2-4].

Compared with the ultimate strain of the reinforcing fibers, the folding strain of continuous-fiberreinforced EMCs is much higher. The main mechanism that allows much higher folding strain without damage is due to microbuckling, and post-microbuckling response of the compressively loaded fibers during bending, which have been confirmed by tests[5-7]. For example, although the ultimate strain of high-performance carbon fibers is usually lower than 1\%, continuous-carbon-fiber-reinforced EMC laminate can receive more than $5 \%$ equivalent bending strain.

Fig. 1 is the sketch of microbuckling in EMC laminate during bending. Microbuckling is regarded as an elastic instability response of the compressively loaded reinforcement fibers. For EMCs under bending, as the temperature is higher than the glass transition temperature of the matrix $\left(T_{\mathrm{g}}\right)$, the matrix will become compliant. The compliant matrix can not supply enough shear stiffness to prevent the fibers from microbulking under the pressure. Therefore the reinforced fibers in the compressive zone can achieve much higher deformation without damage. In the meantime, microbuckles will dramatically decrease the stiffness of the fibers in compressive zone and induce their values to be much lower than that in tensile zone. Since the matrix is compliant and can not endure much higher

\footnotetext{
${ }^{a}$ Corresponding author : 13671108368@126.com
} 
load, the mismatch of the stiffness between the compressive and tensile zones will cause the neutral axis of EMC laminate significantly shifting toward the tensile zone. By such deformation adjustment, the maximum tensile strain of the reinforced fibers can also keep under an endurable value even if the equivalent bending strain of EMC laminate is very high.

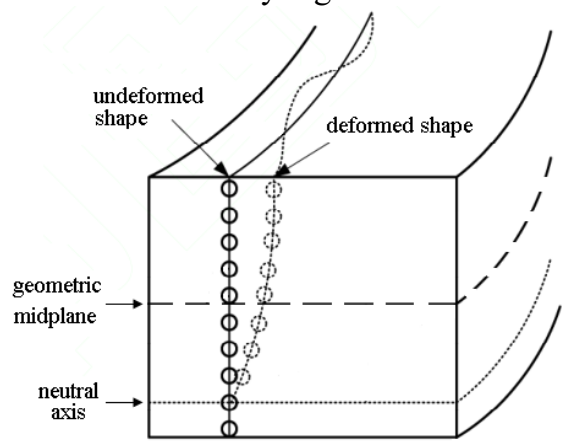

Figure 1. Sketch of microbuckling in EMC laminate during bending.

As an EMC laminate is bent, the prebuckled plate theory can be expressed as

$$
M_{\text {linear }}=\frac{E_{x} t^{3} w \kappa}{12\left(1-v_{x y} v_{y x}\right)}
$$

where $w$ is the plate width, $\kappa$ is the bending curvature, $E_{\mathrm{x}}$ is the longitudinal composite Young's modulus, $t$ is the thickness of the laminate, $v_{\mathrm{xy}}$ and $v_{\mathrm{yx}}$ are respectively the axial, transverse in-plane composite Poisson's ratio. the postbuckled solution given by William H. Francis IV[8] is expressed as the following

$$
M=\frac{G_{x y} t^{2} w}{12 \varepsilon_{b}^{2}}\left[\left(\frac{G_{x y}}{E_{x}}-2 \varepsilon_{b}\right) \sqrt{\left(\frac{G_{x y}}{E_{x}}\right)^{2}+4 \frac{G_{x y}}{E_{x}} \varepsilon_{b}}-\left(\frac{G_{x y}}{E_{x}}\right)^{2}+6 \varepsilon_{b}^{2}\right]
$$

where $G_{\mathrm{xy}}$ is the in-plane composite shear modulus, $\varepsilon_{\mathrm{b}}$ is the equivalent bending strain defined by

$$
\varepsilon_{b}=t \kappa / 2
$$

$\varepsilon_{\mathrm{b}}$ is not the actual strain on either the compression or tension surface because the calculation assumes the neutral strain surface is located at the geometric midplane. However, it is a reasonable indication of the magnitude of the deformation created by the bending. In order to express the moment in terms of the plate curvature rather than the equivalent bending strain, substituting Equation (3) into Equation (2) gives

$$
M=\frac{G_{x y} w}{3 \kappa^{2}}\left[\left(\frac{G_{x y}}{E_{x x}}-t \kappa\right) \sqrt{\left(\frac{G_{x y}}{E_{x x}}\right)^{2}+2 t \kappa \frac{G_{x y}}{E_{x x}}}-\left(\frac{G_{x y}}{E_{x x}}\right)^{2}+\frac{3}{2}(t \kappa)^{2}\right]
$$

The prebuckled plate theory, Equation (1), is valid until the transition curvature at which point the postbuckled solution, Equations (2) or (4), becomes effective. Therefore, this is the point at which microbuckling is predicted to occur. From the point onward, EMC laminate follows the postbuckled solution. The present article aims to provide an analytical solution to predict the transition curvature of EMC laminate under bending. The solution and the intersection point of the straight line given by Equation (1) and the curve given by Equation (4) are compared, and the reasons of errors are also reasonably analyzed.

\section{Analytical solution of transition curvature}

\subsection{Critical microbuckling strain}


By combining the matrix strain energy given by Rosen's solution for shear instability-mode microbuckling composite[9] with Timoshenko's elastic foundation energy[10], a suitable model and solution is proposed to determine the critical wavelength and critical microbuckling strain of the fibermicrobucking in EMC laminate under pure bending. Based on the laboratory observations that the same wavelength of sinusoidal-like and in-plane microbuckling exist in the EMC laminate under pure bending[6-7], the following simplified assumptions are applied: all fibers display sinusoidal-like microbuckles with the same mode number; the microbuckling mode is recognized as a 1D mode instead of 3D mode since no out-of-plane microbuckle is observed in tests; only shear microbuckling is identified as the available tests exhibit that it is the dominated mode for EMC laminates under bending.

Fig. 2 shows the 1D microbuckling mode used in calculating matrix shear energy

$$
U_{m}=\int_{V} \frac{1}{2} G_{m} \gamma_{x y}^{2} d V
$$

where $\gamma_{\mathrm{xy}}$ is the shear strain, and can be determined from Fig. 2 using the following expression

$$
\gamma_{x y}=\frac{d y}{d x}+\frac{h}{2 c} \frac{d y}{d x}
$$

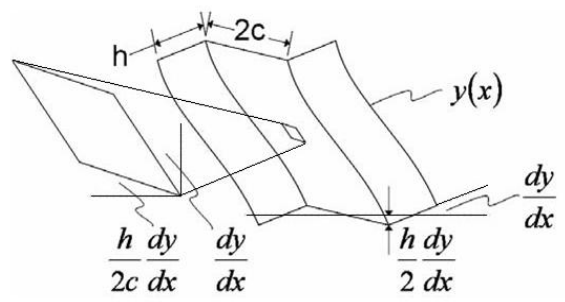

Figure 2. 1D representation of post-microbuckled EMC laminate.

Since a fiber in EMC laminate under bending shows a sinusoidal-like microbuckling shape, it can be described as

$$
y=a \sin (m \pi x / l)
$$

where $m$ is the mode number, namely, number of waves along the fiber length and must be a positive integer to assure the boundary condition, $a$ is the amplitude, and $l$ is the length of the laminate.

Substituting Equation (7) into Equation (6), then Equation (6) into Equation (5) and the integrating result gives the elastic foundation strain energy for specimen with thickness $h$

$$
U_{m}=\frac{G_{m} h c \pi^{2}}{2 l}\left(1+\frac{h}{2 c}\right)^{2} a^{2} m^{2}
$$

where $G_{\mathrm{m}}$ is shear modulus of the matrix, $c$ is half-spacing between adjacent fibers, and $h$ is fiber diameter.

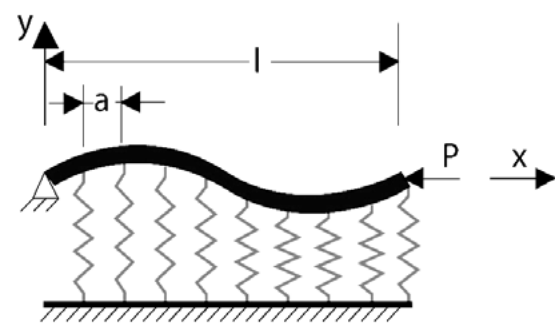

Figure 3. Buckling of a compressively loaded bar on an elastic foundation.

Fig. 3 shows Timoshenko's solution for buckling of a compressively loaded bar in an elastic foundation. The elastic foundation is modeled by a number of springs with a stiffness of $\alpha$ separated by a distance $d$. The stiffness per unit length of the elastic foundation can be expressed as 


$$
\beta=\alpha / d
$$

Therefore the strain energy stored in the elastic foundation is expressed as

$$
U_{s}=\int_{0}^{l} \frac{1}{2} \beta y^{2} d x=\beta l a^{2} / 4
$$

The stiffness $\beta$ of the elastic foundation in a unit length is determined by combining Equations (10) and (8)

$$
\frac{G_{m} h c \pi^{2}}{2 l}\left(1+\frac{h}{2 c}\right)^{2} a^{2} m^{2}=\frac{1}{4} \beta l a^{2}
$$

from which the following expression can be obtained

$$
\beta=\frac{2 h c \pi^{2} G_{m}}{l^{2}}\left(1+\frac{h}{2 c}\right)^{2} m^{2}
$$

The solution for the critical load at which buckling occurs in the mode of Fig. 3 is given by Timoshenko[10] as

$$
P_{c r}=\frac{\pi^{2} E_{f} I_{f}}{l^{2}}\left[m^{2}+\frac{\beta l^{4}}{m^{2} \pi^{4} E_{f} I_{f}}\right]
$$

where $E_{\mathrm{f}}$ is fiber elastic modulus, and $I_{\mathrm{f}}$ is fiber area moment of inertia.

The minimized $P_{\mathrm{cr}}$ is obtained by differentiating Equation (13) with respect to $m$ and the result is

$$
\beta=\frac{\pi^{4} m^{4} E_{f} I_{f}}{l^{4}}
$$

The Equations (14) and (12) yield a new Equation (15):

$$
\frac{2 h c \pi^{2} G_{m}}{l^{2}}\left(1+\frac{h}{2 c}\right)^{2} m^{2}=\frac{\pi^{4} m^{4} E_{f} I_{f}}{l^{4}}
$$

Hence the microbuckling wavelength can be obtained as

$$
\lambda=\frac{l}{m}=\sqrt{\frac{\pi^{2} V_{m} E_{f} h^{3}}{24 c G_{m}}}
$$

where $V_{\mathrm{m}}$ is matrix volume fraction, and it is equal to $2 \mathrm{c} /(2 \mathrm{c}+\mathrm{h})$.

Substituting Equations (14) and (16) into Equation (13), the critical load $P_{\mathrm{cr}}$ can be determined as

$$
P_{c r}=\frac{4 c h G_{m}}{V_{m}^{2}}
$$

Therefore the critical stress and strain are respectively obtained as

$$
\begin{gathered}
\sigma_{c r}=\frac{4 c h G_{m} / V_{m}^{2}}{h(h+2 c)}=\frac{2 G_{m}}{V_{m}} \\
\varepsilon_{c r}=\frac{2 G_{m}}{V_{m} E_{f}}
\end{gathered}
$$

Equation (16) gives a solution of microbuckling wavelength. The critical compressive stress and strain on the initiation of the microbuckle are respectively determined by Equations (18) and (19).

The traditional microbuckling solutions have two fundamental buckling modes, namely shear buckling[9] and extension-buckling[11] modes. The theoretical buckled shape for shear mode is a single half sine function along the length of the specimen of EMC laminate under bending. However, the test specimen is $250 \mathrm{~mm}$ in length and the fibers have a normal wavelength of around $1 \mathrm{~mm}$ [7]. In addition, the critical microbuckling wavelength predicted by the extension mode is about $0.09 \mathrm{~mm}$. Hence, both shear- and extension-mode solutions in the classical fiber microbuckling mechanics are ineffective in forecasting the bending deformation of EMC laminates. Campbell et al.[12] provides a theoretical calculation of microbuckling wavelength in EMC laminates. The wavelength value is 
$1.035 \mathrm{~mm}$, which is in good agreement with the test result, $1 \mathrm{~mm}$. However, according to the expression of microbuckling wavelength the derived critical microbuckling stress goes down with increasing the fiber volume fraction, which is difficult to comprehend and questionable. By assuming a linear relation between the fiber microbuckling amplitude and the thickness of the laminate, Campbell and Maji[13]further proposed an improved 2D microbuckling model. Unfortunately, the similar shortage still exists. Francis et al.[14,8] proposes a model describing the complex postmicrobuckled behavior for EMC laminate under bending. However, this study focuses on postmicrobuckled behavior and the model does not apply for small deformations.

Substituting those test parameters[7] into Equation (16) deduced in this study, the microbuckling wavelength of the continuous-carbon-fiber/resin EMC laminate is about $0.72 \mathrm{~mm}$, which is comparable with the test result, $1 \mathrm{~mm}$. Meanwhile, the expressions of the critical microbuckling stress, Equation (18), and strain, Equation (19), derived from the expression of microbuckling wavelength, Equation (16), in the proposed model are reasonable and intuitively correct.

\subsection{Solution of transition curvature}

As an EMC laminate is bent, it is assumed that it's behavior obeys the lowest energy solution path. the prebuckled laminate theory is valid until the transition curvature at which point the postbuckled solution becomes the lower energy solution. Equation (1) is only applicable to linear laminate and is, thus, referred to as the prebuckled solution to differentiate it from the postbuckled solution provided in Equation (4). Therefore, this is the point at which microbuckling is predicted to occur. From the bending curvature onward, the EMC laminate follows the postbuckled solution.

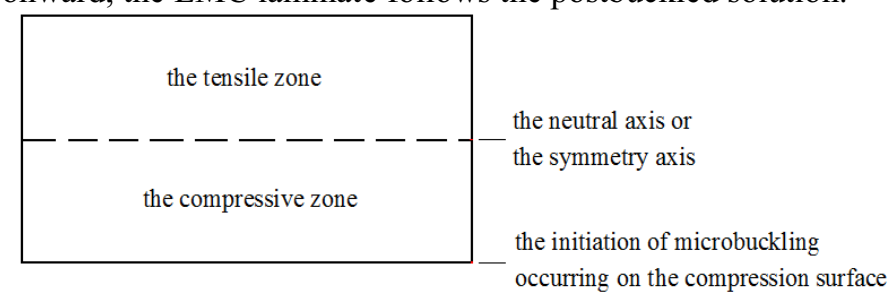

Figure. 4 Sketch of cross section of EMC laminate under transition curvature

Fig. 4 is the sketch of cross section of EMC laminate under the transition curvature. Soft Matrix composite laminate behaves equivalently in tension and compression implying that the neutral axis will reside at the symmetry axis of cross section of laminate. The initiation of microbuckling occurs on the compression surface of the EMC laminate and the other parts of the EMC laminate have not microbuckled. The strain on the compression surface of the EMC laminate can be expressed as

$$
\varepsilon=\frac{t \kappa_{t r}}{2}
$$

where $\kappa_{\mathrm{tr}}$ is the the transition curvature.

Equations (20) and (19) induce a new Equation (21)

$$
\frac{t \kappa_{t r}}{2}=\frac{2 G_{m}}{V_{m} E_{f}}
$$

Hence, the transition curvature of soft matrix composite laminate during bending will be obtained as

$$
\kappa_{t r}=\frac{4 G_{m}}{t V_{m} E_{f}}
$$

The transition curvature, $\kappa_{\mathrm{tr}}$, is proportional to the matrix shear module, $G_{\mathrm{m}}$. $\kappa_{\mathrm{tr}}$ increases with the increase of $G_{\mathrm{m}}$ and decreases with the decrease of $G_{\mathrm{m} .} \kappa_{\mathrm{tr}}$ is inversely proportional to thickness of compoite $t$, matrix volume fraction $V_{\mathrm{m}}$, and fiber Young's modulus $E_{\mathrm{f}}$. Those are understandable and intuitively correct. 


\section{Verification of solution}

Assigning the parameters in Equations (1) and (4) the values given in Table 1 and plotting the moment applied across the laminate length of interest as a function of the bending curvature produces the plot shown in Figure 5. The straight line is given by Equation (1) and the curve is given by Equation (4) in Figure 5. However, due to the absence of a reliable in-plane shear modulus of composite $G_{x y}, G_{x y}$ is treated as a curve fitting parameter and it is found that a value of $G_{\mathrm{xy}}$ which is lower than the value in Table 1 by a factor of 1.9 is needed for data/model correlation[8].

Table 1. Related parameters of soft matrix laminate[8]

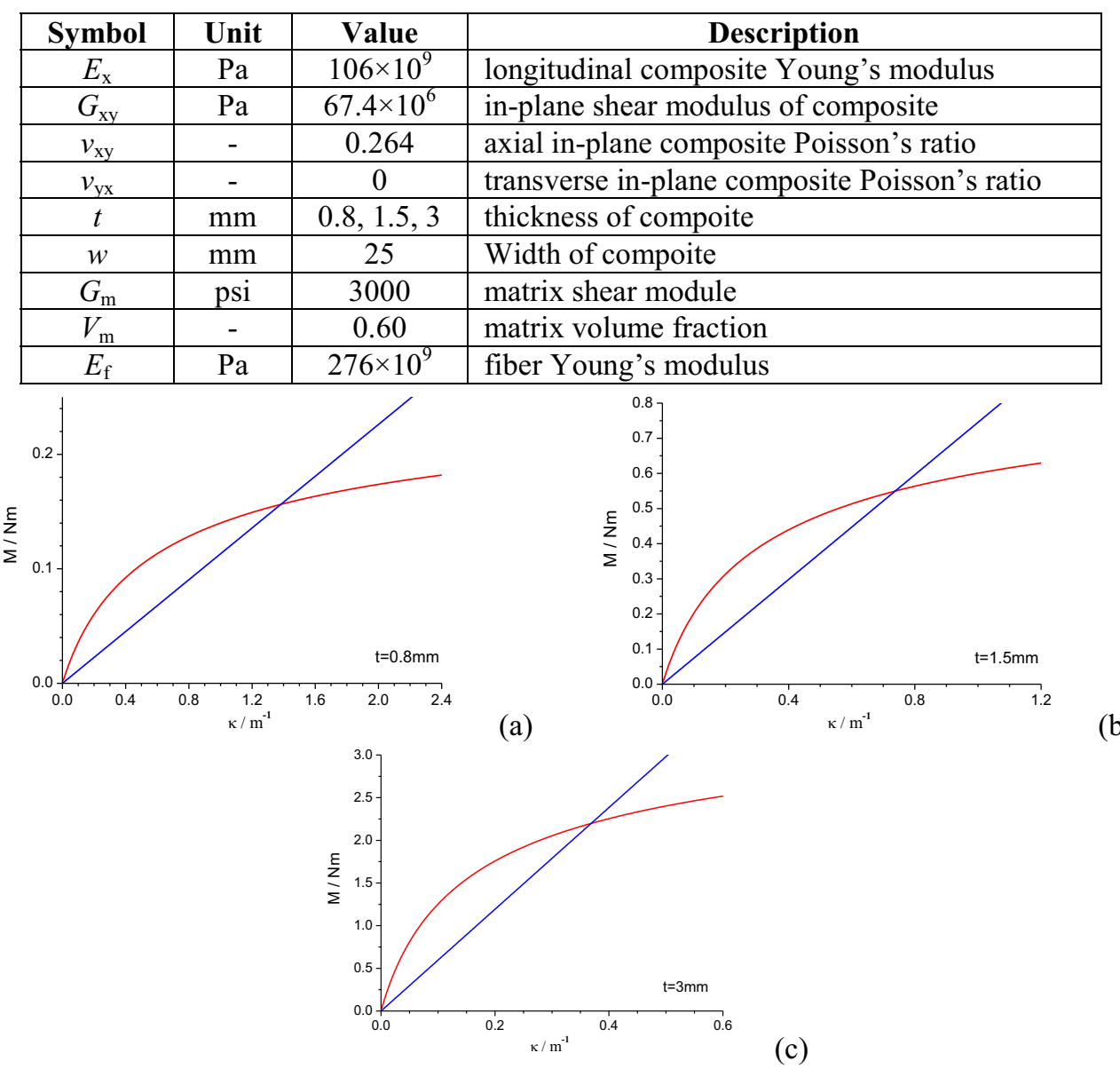

Figure 5. The intersection point of the straight line and the curve (a) $t=0.8 \mathrm{~mm}$, (b) $t=1.5 \mathrm{~mm}$, (c) $t=3 \mathrm{~mm}$

The strain energy stored within the EMC laminate as it is bent is proportional to the area under the curves in Figure 5. Therefore, the lowest energy solution at a given curvature is the solution appearing lowest in the vertical direction. As an EMC laminate is bent, it is assumed that it's behavior follows the lowest energy solution path. Equation (1) is valid until the transition curvature at which point the postbuckled curve becomes the lower energy curve. Therefore, this is the point at which microbuckling is predicted to occur. From the transition curvature onward, the laminate follows the postbuckled solution, Equation (4). From Figure 5, we can know that the intersection points of the straight lines and the curves are respectively about $1.37,0.73,0.36 \mathrm{~m}^{-1}$ under three different thicknesses of laminate $(0.8,1.5,3.0 \mathrm{~mm})$.

In keeping with the requirement of Reference[8], the experiment values will be used to represent the material properties rather than the predicted values and the experiment values usually have higher 
reliability. The value of matrix shear module $G_{\mathrm{m}}$ in Table 1 is not the predicted value given by Reference[8] rather than the value given by Reference[15]. Substituting the relevant data in Table 1 into Equation (22), the results are approximately $0.625,0.333,0.167 \mathrm{~m}^{-1}$ respectively under three different thicknesses of laminate $(0.8,1.5,3.0 \mathrm{~mm})$.

The transition curvatures in Figure 5 are approximately twice the predicted values given by Equation (22). The possible reasons exist in two aspects: (a) devices for measuring hard matrix are now used to measure soft matrix, so the errors are certainly considerable; (b) a literature[6] shows that, the errors may be attributed to the apparent extreme temperature sensitivity of the matrix shear module $G_{\mathrm{m}}$. A temperature of $95^{\circ} \mathrm{C}$ would result in a $G_{\mathrm{m}}$ that is approximately two and a half times the $G_{\mathrm{m}}$ corresponding to $100^{\circ} \mathrm{C}$.

\section{Conclusions}

This paper analyses the transition curvature of soft matrix composite laminates during Bending. By combining Timoshenko's elastic foundation energy with the matrix strain energy given by Rosen's solution for shear-instability-mode microbuckling composite, a model is proposed to describe the microbuckling deformation of a fiber in soft matrix and the critical compressive strain on the initiation of the microbuckle is determined. In the meanwhile, compared with other models, the proposed model is more closed to the real situation. When the strain on the compression surface of the EMC laminate is equal to the critical compressive strain on the initiation of the microbuckle, the analytical solution of the transition curvature is derived. By comparing with the intersection points of the straight lines given by the prebuckled plate theory and the curves given by the postbuckled solution, the solution can be used to predict the point at which microbuckling is predicted to occur, and the causes of the errors are also analyzed in this paper.

\section{Acknowledgement}

This work was funded by Municipal Finance Project of Beijing Municipal Science and Technology Research Institute (PXM2015-178304-000021-008).

\section{References}

1. Lake, M.S., Munshi, N.A. and Tupper, M.L. Application of Elastic Memory Composite Materials to Deployable Space Structures[J]. AIAA Paper No.4602 (2001).

2. Gall, K., Mikulas, M., Munshi, N.A., Beavers, F. and Tupper, M. Development and Testing of a Hinge/Actuator Incorporating Elastic Memory Composites[J]. AIAA Paper No. 1496(2003).

3. Mark, S.L. and Hazelton, C.S. Development of Coilable Longerons Using Elastic Memory Composite Material[J]. AIAA Paper No. 1453 (2002).

4. Francis, W.H., Lake, M.S. and Mallick, K. Development and Testing of a Hinge/Actuator Incorporating Elastic Memory Composites[J]. AIAA Paper No. 1496(2003).

5. Call, K. Micro-mechanisms of Deformation in Fiber Reinforced Polymer Matrix Elastic Memory Composites[J]. AIAA Paper No. 1419(2001).

6. Campbell, D. and Maji, A.K. Deployment precision and Mechanics of Elastic Memory Composites[J]. AIAA Paper No. 1495(2003).

7. Francis, W.H. and Lake, M.S. A Review of Classical Fiber Microbuckling Analytical Solutions for Use with Elastic Memory Composites[J]. AIAA Paper No. 1764(2006).

8. William H. Francis IV. The Mechanics of Post-Microbuckled Compliant-Matrix Composites[D]. Master Thesis. Colorado: University of Colorado, (2008).

9. Rosen, B.W. Fiber Composite Materials[M], American Society for Metals, Metals Park, Ohio, Ch. 3.(1965)

10. Timoshenko, S., Theory of Elastic Stability[M], McGraw-Hill Book Co., Inc., pp109-112.(1936)

11. Dow, N. F., and Rosen, B. W. Evaluations of Filament-Reinforced Composites for Aerospace Applications[J] NASA CR-207(1965). 
12. Campbell, D., Lake, M.S. and Mallick, K. A Study of the Compression Mechanics of Soft-resin Composites[C] In: The 45th AIAA/ASME/ASCE/AHS/ASC Structures, Structural Dynamics and Materials Conference, Palm Springs, California.(2009)

13. Campbell, D. and Maji, A.K. Failure Mechanisms and Deployment Accuracy of Elastic Memory Composites[J]. Journal of Aerospace Engineering, 19(3):184-193.(2006)

14. Francis, W.H., Campbell, D., Lake, M., Dunn, M. and Qi, H.J. Elastic Memory Composite Microbuckling Mechanics: Closed-Form Model with Empirical Correlation[J]. AIAA Paper No.2164(2007).

15. Douglas Campbell, Mark S Lake, Kaushik Mallick. A study of the bending mechanics of elastic memory composites [C]. 45th AIAA/ASME/ASCE/AHS/ASC Structures, Structural Dynamics \& Materials Conference19-22 April 2004, Palm Springs, California.(2009) 